\title{
Une exception française
}

La scolarisation de la formation professionnelle initiale

Another French exception. Initial professional training

Una excepción francesa. La escolarización de la formación profesional inicial

\section{Vincent Troger}

\section{OpenEdition}

1 Journals

Édition électronique

URL : https://journals.openedition.org/ries/1722

DOI : $10.4000 /$ ries. 1722

ISSN : 2261-4265

Éditeur

France Education international

\section{Édition imprimée}

Date de publication : 1 décembre 2003

Pagination : 117-126

ISBN : 978-2-8542-0558-9

ISSN : $1254-4590$

\section{Référence électronique}

Vincent Troger, « Une exception française », Revue internationale d'éducation de Sèvres [En ligne], 34 I décembre 2003, mis en ligne le 23 novembre 2011, consulté le 06 juillet 2021. URL : http:// journals.openedition.org/ries/1722 ; DOI : https://doi.org/10.4000/ries.1722

(c) Tous droits réservés 


\section{Une exception française}

\section{La scolarisation de la formation professionnelle initiale}

\section{Vincent Troger}

À la fin du cycle de scolarité obligatoire, qui s'accomplit de six à seize ans à l'école primaire puis au collège, les jeunes Français ont le choix entre trois orientations différentes : continuer des études au lycée, entrer dans un lycée professionnel ou devenir apprenti dans une entreprise. Ensuite, pour ceux qui ont choisi le lycée, une seconde orientation est proposée à la fin de la première année (classe de seconde) : continuer des études générales ou choisir une filière technologique.

Si on examine la répartition des flux d'élèves après ces deux paliers d'orientation, à la fin du collège obligatoire et après la première année de lycée, on s'aperçoit que les élèves qui s'orientent ou sont orientés vers les lycées professionnels dès la sortie de la scolarité obligatoire représentent environ $30 \%$ d'une classe d'âge et ceux qui après un an de lycée choisissent de poursuivre des études technologiques représentent environ $15 \%$ de leur classe d'âge. Autrement dit, 45 \% des jeunes Français poursuivent une formation professionnelle initiale au sein d'établissements de l'enseignement secondaire, ce qui représente un peu moins de la moitié des lycéens. On peut exprimer cette réalité d'une autre manière : si plus de $60 \%$ des jeunes Français obtiennent aujourd'hui un baccalauréat, la moitié de ces bacheliers sont en fait titulaires de baccalauréats professionnels ou technologiques qu'ils ont dans leur grande majorité préparés dans un établissement scolaire. La comparaison de ces données avec la proportion de jeunes qui choisissent l'apprentissage à la fin de la scolarité obligatoire suffit à illustrer une des caractéristiques fondamentales de notre système de formation professionnelle initiale : ils ne sont qu'environ $8 \%$ à signer un contrat d'apprentissage et à apprendre un métier dans une entreprise. La très grande majorité des jeunes qui s'orientent vers une formation professionnelle initiale après la scolarité obligatoire le font donc au sein d'établissements scolaires, pour la plupart publics.

Dans beaucoup d'autres pays d'Europe, il existe des établissements équivalents à nos lycées professionnels ou technologiques mais dans aucun d'entre eux ces établissements n'assument une part aussi déterminante de la formation professionnelle initiale. Il convient donc de s'interroger sur cette particularité française. Dans un premier temps, nous rappellerons les origines historiques de cette scolarisation massive de la formation professionnelle initiale. La seconde partie de ce texte sera consacrée au fonctionnement actuel 
des formations professionnelles scolarisées, essayant notamment d'élucider la nature des demandes sociales dont ces formations font l'objet ainsi que le mode de relation qu'elles entretiennent avec les entreprises. La conclusion tentera de proposer quelques hypothèses prospectives.

\section{LES CHOIX FONDATEURS ${ }^{1}$}

Les enseignements techniques et professionnels se sont constitués en France par l'alliance de l'État et du patronat des industries modernes. En 1963, un universitaire anglais n'hésitait pas à parler, à propos de l'économie française, d'une «conspiration entre l'État modernisateur et le grand capital » ${ }^{2}$. Même si la formule était volontairement, et à plusieurs titres, provocatrice, il n'en reste pas moins qu'elle pourrait en partie rendre compte de l'histoire des enseignements technologiques et professionnels français.

L'enseignement technologique tel qu'il existe aujourd'hui dans les lycées français est en effet né des initiatives des premiers gouvernements républicains à la fin du $\mathrm{XIX}^{\mathrm{e}}$ siècle. Ces gouvernements avaient alors une double préoccupation : d'une part ancrer la légitimité du régime républicain dans l'opinion publique, particulièrement en poursuivant l'éducation de la jeunesse populaire au-delà de l'école primaire, d'autre part répondre aux besoins de formation des industries les plus modernes, notamment mécaniques et métallurgiques : leur production était en effet essentielle, à la fois pour la puissance économique du pays mais aussi pour sa puissance militaire. «Oui messieurs, sur le champ de bataille industriel comme sur l'autre les nations peuvent tomber et périr [...]. C'est à ce grand danger que doit parer l'enseignement professionnel dans notre pays [...]; à l'heure qu'il est messieurs, relever l'atelier c'est relever la Patrie», proclame Jules Ferry en inaugurant le chantier de la première école professionnelle d'État à Vierzon en $1883^{3}$. Une partie des élites politiques républicaines et une partie du patronat vont donc, dès cette époque, s'allier pour former ce que des historiens anglais qui ont étudié cette question ont appelé un lobby ${ }^{4}$.

Ce lobby fait alors pression en faveur de deux objectifs. Le premier est le développement d'écoles techniques financées conjointement par l'État et les collectivités locales. Presque trois cents établissements sont ouverts pendant les trois premières décennies du $\mathrm{XX}^{\mathrm{e}}$ siècle. Les actuelles filières technologiques des

1. Certaines parties de ce chapitre sont empruntées à un texte publié par l'auteur dans Sciences Humaines, $\mathrm{n}^{\circ} 140$, juillet 2003.

2. Shonfield A., Modern capitalism. The changing balance of public and private power, Oxford University Press, 1963.

3. Cité par Troger V., "L'identité perdue des enseignements techniques et professionnels», in Moreau G., Les patrons, l'État et la formation des jeunes, La Dispute, 2002.

4. R. Fox and G. Weisz, The organization of science and technology in France, 1908-1914, Cambridge University Press, 1980. 
lycées sont les héritières directes de ces écoles : devenues en 1959 les lycées techniques, elles ont été complètement intégrées administrativement à l'enseignement secondaire en 1985. Ce premier objectif a donc été atteint mais le public concerné est demeuré restreint par rapport à l'ensemble des besoins de main-d'œuvre qualifiée.

Le second objectif est la mise en place d'une législation visant à réglementer la formation de l'ensemble des ouvriers et des employés, afin de reconnaître et de favoriser les efforts des entreprises qui acceptaient d'en assumer les coûts. Après la création en 1919 d'un diplôme qui sanctionne la qualification professionnelle, le certificat d'aptitude professionnelle (CAP), des cours de formation théorique sont rendus obligatoires pour les apprentis (loi Astier) et, en 1925, une taxe d'apprentissage est imposée aux entreprises qui ne forment pas d'apprentis. Explicitement inspirées de l'exemple allemand, ces mesures visaient à réguler la formation de la main-d'œuvre qualifiée en obtenant une mutualisation des initiatives des entreprises en matière de formation. Mais ce second objectif n'a pas pu être globalement atteint, malgré les efforts de certaines grandes entreprises, notamment dans la construction mécanique et électrique ou dans les banques.

Cette réticence de la majorité des entreprises à participer à l'effort de formation explique le développement de l'enseignement professionnel. En effet, d'abord dans la France occupée et dirigée par le gouvernement de Vichy, puis après la Libération, un certain nombre d'initiatives vont occuper un terrain laissé en quelque sorte en friche par l'échec des efforts antérieurs en faveur d'une prise en charge collective de l'effort de formation par les entreprises.

Poursuivant un rêve anachronique et ultraconservateur de restauration du système des corporations, le gouvernement de Philippe Pétain va légiférer de manière décisive en faveur du développement d'une formation professionnelle plus étroitement contrôlée par l'État : il établit un monopole d'État sur la délivrance des diplômes professionnels, tandis qu'une loi de 1942 élabore une classification des qualifications ouvrières en introduisant dans leur définition la référence au CAP. Simultanément, il installe sur tout le territoire des centres de formation professionnelle destinés à la fois à assurer l'encadrement idéologique de la jeunesse ouvrière et à former des travailleurs manuels qualifiés. En 1944, plus de huit cents établissements de ce type scolarisent à plein temps environ cinquante mille élèves. Vichy lègue ainsi aux gouvernements de la Libération à la fois un nouveau réseau d'écoles professionnelles et un dispositif législatif qui instaure une équivalence entre un diplôme préparé à l'école, une qualification reconnue sur le marché du travail et un salaire.

Dans un contexte idéologique opposé, les gouvernements de la Libération vont reprendre et généraliser ce dispositif. La grande majorité des nouvelles élites politiques du pays sont alors converties, comme à peu près 
partout en Europe, aux nouveaux principes économiques dominants, keynésiens ou socialistes, qui prônent l'intervention de l'État. En outre, la conjonction d'un creux démographique dans les générations en âge de travailler et de besoins considérables de main-d'œuvre qualifiée, liés à l'effort de reconstruction, ne laisse pas aux entreprises une large marge de manœuvre pour assumer la formation professionnelle. Il y a donc consensus, notamment des syndicats et du grand patronat, autour du développement des formations professionnelles scolarisées sous le contrôle de l'État : les centres de formation professionnelle hérités de Vichy accueillent deux cent mille élèves en 1959, au moment où ils deviennent collèges d'enseignement technique (CET) ${ }^{5}$. Simultanément, les écoles techniques antérieurement créées par la troisième République passent de soixante dix mille à cent quarante mille élèves et deviennent des lycées techniques.

La dernière étape de ce processus de scolarisation de la formation professionnelle initiale participe de la modernisation du pays voulue par le général De Gaulle de retour au pouvoir en 1958. Persuadé de la nécessité, en France comme dans tous les pays comparables, d'élever le niveau de qualification de la population pour accroître la puissance économique nationale, l'entourage du général De Gaulle met en œuvre une politique de croissance et de prolongement de la scolarisation. Les enseignements technique et professionnel sont alors largement développés, mais par souci de rationalisation et de contrôle des coûts, ils sont intégrés à l'enseignement secondaire, dont ils deviennent des filières ${ }^{6}$. Plusieurs nouveaux diplômes sont créés (brevets d'enseignement professionnel, baccalauréats technologiques, brevets de techniciens supérieurs) qui sanctionnent chaque niveau d'études professionnelles ou technologiques. Dans le même esprit de gestion rationalisée, des experts imaginent d'établir une correspondance théoriquement rigoureuse entre les parcours de formation, les diplômes qui les sanctionnent et l'insertion dans les emplois. En 1965, le commissariat général au plan met ainsi au point une nomenclature des niveaux de diplômes (de I à VI) censés correspondre à des niveaux de qualification précis sur le marché du travail. C'est notamment de cette époque que date l'habitude très française de faire apparaître dans les annonces d'emploi la référence à un niveau précis de diplôme $(b a c+2$, bac +3 , etc. $)^{7}$.

5. Les centres de formation professionnelle ouverts par Vichy sont devenus centres d'apprentissage en 1944, CET en 1959, LEP en 1976 et lycées professionnels en 1985.

6. Ce qui se traduit notamment par la suppression, au ministère de l'Éducation nationale, d'une direction autonome de l'enseignement technique.

7. Pour plus d'information sur la dimension historique de la formation professionnelle scolarisée, on peut consulter: Brucy G., Histoire des diplômes de l'Enseignement technique et professionnel (1880-1965). L'État, l'école, les entreprises et la certification des compétences, Paris, Belin, 1998, et Pelpel P. et Troger V., Histoire de l'Enseignement technique, Paris, L'Harmattan, 2002 (1 éd. Hachette, 1993). 


\section{UN BILAN CONTRASTÉ}

En un peu moins d'un siècle, l'action de l'État, soutenue par une partie du grand patronat, a donc abouti à ce que la formation professionnelle initiale française soit massivement assumée par le système scolaire public, en se fondant sur des formations sanctionnées par des diplômes reconnus sur le marché du travail. Cette évolution autorise le sociologue Eric Verdier ${ }^{8}$ à parler d'un mode de régulation de la relation formation-emploi qui repose sur une "convention méritocratique» : le diplôme scolaire, délivré par l'État, est perçu comme le seul repère susceptible de garantir une compétition équitable entre les individus pour l'accès à l'emploi.

On ne peut cependant s'en tenir à ce constat général pour rendre compte du fonctionnement de ce système. Si le principe général de «convention méritocratique» dont parle Eric Verdier demeure globalement valide, la réalité $\mathrm{du}$ fonctionnement des enseignements technologiques et professionnels est plus complexe et plus conflictuelle qu'il n'y paraît au premier regard. Pour aller vite, et pour tenter un bilan du fonctionnement actuel de la formation professionnelle initiale en France, on peut en retenir trois principales caractéristiques.

\section{Une pédagogie reconnue}

La scolarisation par l'État n'a en effet pas rompu les relations entre les enseignements professionnels ou technologiques et les entreprises. Ces relations sont au contraire entretenues à trois niveaux. Au ministère d'abord, où les représentants des entreprises, des syndicats de salariés et de l'Éducation nationale entretiennent des relations régulières au sein des commissions professionnelles consultatives (CPC). Ces commissions élaborent des référentiels d'emplois et de formation qui servent de base pour la conception des programmes et des diplômes technologiques. Ces diplômes sont régulièrement rénovés à la demande des représentants des employeurs. C'est par exemple à leur demande qu'à été conçu en 1985 un nouveau diplôme, le baccalauréat professionnel, qui tend aujourd'hui à remplacer le CAP sur certains segments du marché du travail. Mais les relations avec les entreprises sont aussi entretenues directement par les corps d'inspection de l'enseignement professionnel et technologique et par les enseignants : une proportion importante d'entre eux a exercé le métier qu'ils enseignent avant de devenir professeurs ${ }^{9}$. Ils conservent donc avec leurs professions d'origine, soit formellement à travers des activités associatives, soit de manière informelle, des relations relativement étroites. Enfin, au niveau des établissements, les relations directes avec les entreprises existent pour deux raisons. La première est le versement de la taxe d'apprentissage :

8. Verdier E., "La France a-t-elle changé de régime d'éducation et de formation ? », Formation-Emploi, nº 76, 2001. 9. Selon les secteurs professionnels, la proportion d'enseignants issus du monde du travail est très variable, mais elle est rarement inférieure à $50 \%$. 
cette taxe peut en effet être payée directement par les entreprises aux établissements de leur choix et beaucoup de chefs d'établissements obtiennent qu'elle leur soit versée en offrant des services aux employeurs (expérimentation de nouveaux matériels, formation continue des personnels, ouvertures de formations initiales adaptées aux besoins des entreprises...). La seconde est l'organisation des stages des élèves. Ces stages sont désormais validés pour l'attribution de la plupart des diplômes technologiques, ce qui oblige les professeurs à rechercher les entreprises ou les administrations qui acceptent d'accueillir leurs élèves, et donc à entretenir avec elles des relations suivies et à connaître leurs exigences.

Les avantages de ce système sont connus. Il permet de capitaliser une expérience de la pédagogie des formations professionnelles initiales qui n'est pas dépendante des fluctuations de la situation économique ou des cultures particulières d'entreprise. L'exemple anglais montre a contrario que lorsque des pans entiers de l'industrie disparaissent, les savoirs pédagogiques dont les entreprises de ces secteurs étaient porteuses à travers la pratique de l'apprentissage disparaissent avec elles. Si les besoins en formation dans ces secteurs réapparaissent, ou s'ils continuent d'exister dans d'autres secteurs de l'industrie, la formation professionnelle est alors à réinventer, ce qui est actuellement le cas au RoyaumeUni. En Allemagne, le système d'apprentissage collectivement pris en charge par les entreprises demeure performant, mais il est lui aussi très dépendant de la conjoncture économique, tandis que les formations délivrées sont souvent limitées aux pratiques spécifiques de l'entreprise qui les assume, ce qui ne facilite pas la mobilité de la main-d'œuvre qualifiée. En règle générale, les élèves formés par l'enseignement professionnel et technique français, qui ont reçu une formation plus globale, sont certes moins immédiatement opérationnels lorsqu'ils intègrent une entreprise, mais sont en revanche mieux préparés à évoluer dans leur emploi et à s'adapter à des cultures techniques différentes. En outre, l'un des avantages de la certification des formations par des diplômes nationaux est de permettre aux employeurs de connaître à l'avance, en tout lieu du territoire national, les caractéristiques de la main-d'œuvre qu'ils souhaitent embaucher.

\section{Des critiques sévères}

Seconde caractéristique dominante, ce système a été sévèrement critiqué au cours des vingt dernières années, essentiellement pour deux raisons. La première est que les jeunes issus des formations scolarisées sont peu préparés à se plier aux exigences comportementales que les nouvelles conditions de production imposent désormais aux salariés. Pour aller vite, le système de production de biens et de services a été de plus en plus déterminé par l'exigence de qualité. Ce sont de moins en moins les contraintes matérielles de la production qui déterminent l'organisation du travail et de plus en plus les exigences des clients. Dans les entreprises, les services commerciaux et de marketing dictent désormais leurs exigences aux services de production, alors que la situation 
était inverse il y a trente ans. De la même manière, dans les services publics, très développés en France, on parle de moins en moins des usagers, et de plus en plus des clients. Cette évolution signifie pour les ouvriers, les employés et les cadres intermédiaires, que l'on exige d'eux des compétences nouvelles qui s'ajoutent aux qualifications techniques qu'ils doivent toujours posséder. On leur demande de mieux communiquer, d'être capables de s'adapter rapidement à des situations nouvelles, d'être capables d'assumer une partie de la relation avec la clientèle, etc. ... La formation professionnelle scolarisée a su s'adapter aux évolutions technologiques, mais elle est moins apte à transmettre les qualités comportementales désormais attendues des salariés. Pour le dire autrement, les élèves des lycées professionnels et des filières technologiques de lycées sont moins «dociles» que ceux formés par l'apprentissage.

Des critiques d'un autre type ont été formulées par des milieux patronaux représentant les petites entreprises, particulièrement dans les secteurs artisanaux. Nombreux dans le bâtiment ou l'alimentation, ces employeurs se plaignent souvent de la dimension trop généraliste des formations délivrées par les enseignements technologiques et professionnels. Dans ces secteurs, où les pratiques professionnelles demeurent marquées par une forte dimension manuelle, et où l'apprentissage du métier nécessite plus une mémorisation de gestes et de techniques qu'une culture technologique, le modèle dominant de la formation scolarisée apparaît parfois décalé avec la réalité de l'exercice de la profession. Depuis une dizaine d'années, notamment sous l'impulsion des régions qui ont acquis depuis 1993 un pouvoir décisionnel en matière de formation professionnelle, l'apprentissage a connu un renouveau dans ce type de métiers. Il s'est également développé à des niveaux de qualification supérieurs, correspondant aux formations technologiques post-baccalauréat. Il touche souvent des publics qui préfèrent l'apprentissage aux formations scolarisées, à la fois en raison des rémunérations qui sont offertes et parce qu'ils en espèrent, souvent à juste titre, une insertion plus rapide sur le marché du travail.

\section{Une position de relative infériorité au sein du système}

Pour terminer ce bref tableau de la situation de la formation professionnelle scolarisée en France, il faut enfin en souligner une des dimensions essentielles, qui constitue la troisième caractéristique que nous voulions mettre en évidence : il s'agit de sa position de relative "infériorité» dans le système éducatif. Ce discrédit des enseignements technologiques et surtout professionnels a principalement deux origines.

La première d'entre elles est la dévalorisation du travail manuel qui a accompagné la diminution de la population ouvrière, le chômage et la tertiarisation des emplois. Laminée par la désindustrialisation des grandes régions 
ouvrières traditionnelles du Nord et de l'Est, par l'arrivée des femmes et des immigrés dans les emplois non-qualifiés, par le développement des nouvelles technologies, la culture ouvrière traditionnelle, fondée sur la solidarité et l'éthique du travail, ne s'est maintenue que dans de rares secteurs de l'industrie. Les enfants des milieux populaires ne se reconnaissent plus dans l'image de l'ouvrier habile, dur au mal et solidaire de ses camarades. Au contraire, ils veulent fuir cet univers si étranger à l'image moderne du travail que symbolisent désormais le bureau, l'écran d'ordinateur ou les grands magasins. Les filières les plus demandées des lycées professionnels et de l'enseignement technologique des lycées sont ainsi les filières tertiaires, alors qu'elles offrent moins de possibilités de carrière que les filières industrielles, et que certaines conduisent même aux emplois aujourd'hui les plus précaires et les plus prolétarisés du marché du travail, notamment dans la grande distribution.

La deuxième raison de la marginalisation des filières professionnelles et technologiques au sein du système éducatif peut être comprise comme un effet pervers de l'extension de la scolarisation. La multiplication des écoles d'ingénieurs, des écoles commerciales et le développement de l'enseignement technologique universitaire fournissent de plus en plus directement aux entreprises et à l'administration les cadres et les cadres supérieurs dont elles ont besoin. Or l'efficacité de ce système a une conséquence négative pour les diplômés de l'enseignement technique et surtout professionnel : elle rend de plus en plus difficile pour les ouvriers ou les employés subalternes l'accès aux emplois supérieurs par la voie de la promotion interne. Les emplois de niveaux supérieurs sont de plus en plus occupés directement par les diplômés des écoles d’ingénieurs, de commerce, ou de l'université. Choisir l'enseignement technique, et surtout professionnel, c'est prendre le risque de rester enfermé pour toute la durée de l'existence dans une condition socioprofessionnelle subalterne ou au mieux intermédiaire.

Ce blocage a évidemment été aggravé par la croissance dramatique du chômage après la crise pétrolière de 1975. À partir de la fin des années soixante-dix, les enseignements techniques et professionnels, malgré leur croissance numérique, sont ainsi assez largement considérés comme des «filières de relégation » du système scolaire. Les familles tentent systématiquement d'éviter ces filières et refusent majoritairement les propositions d'orientation allant en ce sens, surtout pour l'enseignement professionnel. De ce fait l'enseignement professionnel n'accueille plus que les élèves ayant terminé la scolarité obligatoire au collège en situation d'échec, et les filières technologiques accueillent les élèves qui, en fin de seconde, ont un niveau insuffisant pour poursuivre en enseignement général littéraire, scientifique ou économique. Le sociologue Pierre Bourdieu a désigné les élèves de l'enseignement technologique comme les « exclus de l'intérieur » du système éducatif ${ }^{10}$.

10. Bourdieu Pierre, Champagne Philippe, «Les exclus de l'intérieur », in Bourdieu Pierre, La misère du monde, Le Seuil, 1995 . 


\section{VERS UN RAPPROCHEMENT ENTRE FORMATION SCOLARISÉE ET APPRENTISSAGE?}

Cette conjonction des critiques issues des milieux patronaux et d'une marginalisation de fait au sein du système éducatif a évidemment conduit le ministère à chercher à « revaloriser» les enseignements professionnels et technologiques et donc à en transformer les structures. Des expérimentations d'établissements offrant à la fois des formations scolaires, des formations complémentaires à l'apprentissage et des formations d'adultes ont été encouragées sous le nom de «lycées des métiers ». Il est probable qu’à terme le rapprochement entre les modalités de formation scolaire et les modalités de formation d'apprentis ou d'adultes se rapprocheront. La pratique systématique des stages en entreprises dans les lycées professionnels est un des signes tangibles de cette évolution, et les pressions de la Commission européenne en faveur de la «formation tout au long de la vie» ne peuvent que l'encourager. On sait néanmoins qu'elle sera nécessairement longue, pour au moins trois raisons. D'une part, les entreprises françaises ont pris l'habitude de faire supporter l'essentiel de l'effort de formation professionnelle initiale au système scolaire et tout indique qu'elles ne sont pas prêtes à y renoncer. D'autre part, les personnels des enseignements technologiques, des lycées professionnels, des centres de formation d'apprentis et des formations d'adultes n'ont pas les mêmes statuts et ne dépendent pas des mêmes ministères ${ }^{11}$. Une fusion de ces formations supposerait donc de longues négociations avec les syndicats respectifs de ces personnels, et entre eux. Enfin, et surtout, on a vu que les enseignements technologiques et professionnels servent en quelque sorte de soupape de sécurité au système éducatif français : ils accueillent les $45 \%$ d'élèves qui ont plus ou moins échoué au cours de leur scolarité obligatoire. Une évolution rapide du statut des formations professionnelles initiales supposerait donc une double transformation : celle des pratiques des entreprises en matière de formation et celle des pratiques pédagogiques du collège. Ces transformations sont en cours, mais elles touchent trop profondément à certains des fondements traditionnels de la société française pour qu'elles puissent aboutir à court terme.

\section{BibLIOGRAPHIE}

AGULHON C. (1994): L'enseignement professionnel. Quel avenir pour les jeunes? Paris, Les Éditions de l'Atelier, 265 p.

\footnotetext{
11. Les professeurs de lycées professionnels et les professeurs des filières technologiques de lycées sont tous fonctionnaires, mais ils ne sont pas recrutés par le même concours et n'ont pas le même statut. Les personnels des centres de formations d'apprentis (CFA) ont un autre statut dans les centres publics et sont sous contrat privé lorsqu'ils travaillent dans les CFA privés, les plus nombreux. Il en va de même pour les formateurs d'adultes. Enfin, les formations d'adultes sont sous la tutelle du ministère du Travail et de l'Emploi et les lycées professionnels agricoles sous celle du ministère de l’Agriculture.
} 
BEAUD S. (septembre 1996) : «Les «bacs pro». La désouvriérisation du lycée professionnel», Actes de la recherche en sciences sociales, ${ }^{\circ} 114, \mathrm{pp}$. 21-29.

BRUCY G. (1998): Histoire des diplômes de l'enseignement technique et professionnel (1880-1965). L'État, l'école, les entreprises et la certification des compétences, Paris, Belin.

CHARLOT B., FIGEAT M. (1985): Histoire de la formation des ouvriers, 1789-1984, Paris, Minerve, 601 p.

CHARMASSON T., LELORRAIN A.M., RIPA Y. (1987): «L'enseignement technique de la Révolution à nos jours ", Paris, INRP-Economica, 783 p.

JOBERT A., MARRY C., TANGUY L. (1995) : Éducation et travail en GrandeBretagne, Allemagne et Italie, Paris, A. Colin, 395 p.

LEGOUX Y. (1972): Du compagnon au technicien (l'école Diderot et l'évolution des qualifications), 1873-1972, Technique et vulgarisation.

MOREAU G. (dir.) (2002): Les patrons, l'État et la formation des jeunes, La Dispute.

PELPEL P. et TROGER V. (2002) : Histoire de l'enseignement technique, Paris, L'Harmattan ( $1^{\text {re }}$ éd. Hachette, 1993).

TANGUY L. (1991): Quelle formation pour les ouvriers et les employés en France?, Paris, La Documentation française, 142 p.

TROGER V. (coord.) : Les formations professionnelles entre l'école et l'entreprise, Revue Française de Pédagogie n 131, avril-mai-juin 2000. 